\title{
Molecular Clustering of Skeletal and Cardiac Ryanodine Receptors
}

\author{
Joshua Lobo $^{1}$, Sonali Dhindwal ${ }^{1}$ and Montserrat Samsó ${ }^{1}$ \\ 1. Department of Physiology and Biophysics, Virginia Commonwealth University, Richmond, VA, USA.
}

Contraction of skeletal and cardiac muscles is triggered by transient increase of cytosolic $\mathrm{Ca}^{2+}$ concentration. Such $\mathrm{Ca}^{2+}$ transient is originated from the massive release of $\mathrm{Ca}^{2+}$ stored in the sarco/endoplasmic reticulum (SR), via intracellular $\mathrm{Ca}^{2+}$ channels known as the ryanodine receptors (RyRs). Functional $\mathrm{Ca}^{2+}$ release units formed by discrete clusters of multiple RyRs open synchronously upon activation of plasmalemmal voltage-gated $\mathrm{Ca}^{2+}$ channels. A different subcellular anatomy of the cardiac and skeletal muscle cells, and two different RyR isoforms (RyR1 in skeletal muscle and RyR2 in heart), result in fundamentally different temporal and spatial $\mathrm{Ca}^{2+}$ release profiles $[1,2]$.

The cytoplasmic domain of the RyR has a characteristic square shape of approximately $30 \mathrm{~nm}$ side that can be distinguished in situ by TEM. In skeletal muscle, RyR1s assemble in a checkerboard-like fashion on the edge of the terminal cisternae of the SR, forming two rows, whereby the site of inter-RyR1 molecular contact was assigned to the helical domains of two neighboring RyR1s [3]. In heart, RyR2s form clusters of variable size and dimensions, with two forms of arrangement: checkerboard-like and lineal side-by-side, besides single RyR2s [4]. For the checkerboard-like orientation, it was assumed that RyR2s had a similar organization than that found in the RyR1 arrays. However, this was not firmly established because the low resolution of electron tomography, thin section, or rotary shadowing was insufficient to discern substructure within RyRs in situ.

To ascertain the domains involved in RyR dimerization in the cardiac isoform, we carried out a statistical analysis on RyR2s assembled in vitro. RyR2s were purified from heart tissue, negatively stained and imaged by TEM at $200 \mathrm{kV}$ and a magnification of 50,000x. Hundreds of pairs of interacting RyR2s were selected and processed using single-particle image processing software. The RyR2 pairs were aligned using a reference-free strategy and then classified, yielding 2D class averages. Averages where at least one of the RyR2s in the pair did not have substructure were discarded, as they reflect RyR2s next to each other by chance. 2D class averages where both RyR2s in the pair had substructure correspond to the average of multiple pairs of RyR2s with a fixed position between them. These 2D averages enabled determining the absolute orientation of interacting RyR2s. The analysis was repeated in two independent datasets with reproducible $2 \mathrm{D}$ class averages. In both analyses, there were two types of rigidly connected RyR2s: side-by-side (15\%), and a configuration initially categorized as checkerboard-like $(\sim 30 \%)$. Surprisingly, the checkerboard-like interaction was not through the helical domains as previously assumed, but through the SPRY1 domain, which is a domain with known scaffolding properties. Furthermore, close examination revealed that the array geometry diverges from the checkerboard geometry, and consists of non-orthogonal (oblique) arrays with an average of $12^{\circ}$ angle between neighboring channels [5] (Fig. 1).

Our analogous analysis on the RyR1 isoform confirms a true checkerboard configuration as previously reported, with interaction through the helical domains. This suggests that the two RyR isoforms, with high degree of homology, have different dimerization possibilities, which account for the substantially different quaternary arrangements observed (Fig. 1). We place this finding in the context of the cryoEM 3D structure of RyR (Fig. 2), and propose an evolutionary basis underlying the different modes of 
dimerization.

References:

[1] SQ Wang et al. Proc Natl Acad Sci USA 101 (2004), 3979.

[2] A Gonzalez et al. Proc Natl Acad Sci USA 97 (2000), 4380.

[3] DG Ferguson, HW Schwartz and C Franzini-Armstrong. J Cell Biol 99 (1984), 1735.

[4] P Asghari et al. Protoplasma 249 Suppl 1 (2012), S31.

[5] V Cabra, T Murayama and M Samsó. Biophys J 110 (2016), 2651.

[6] Supported by grants NIH AR068431, American Heart Association 14GRNT19660003, and Muscular Dystrophy Association MDA352845 (to MS).
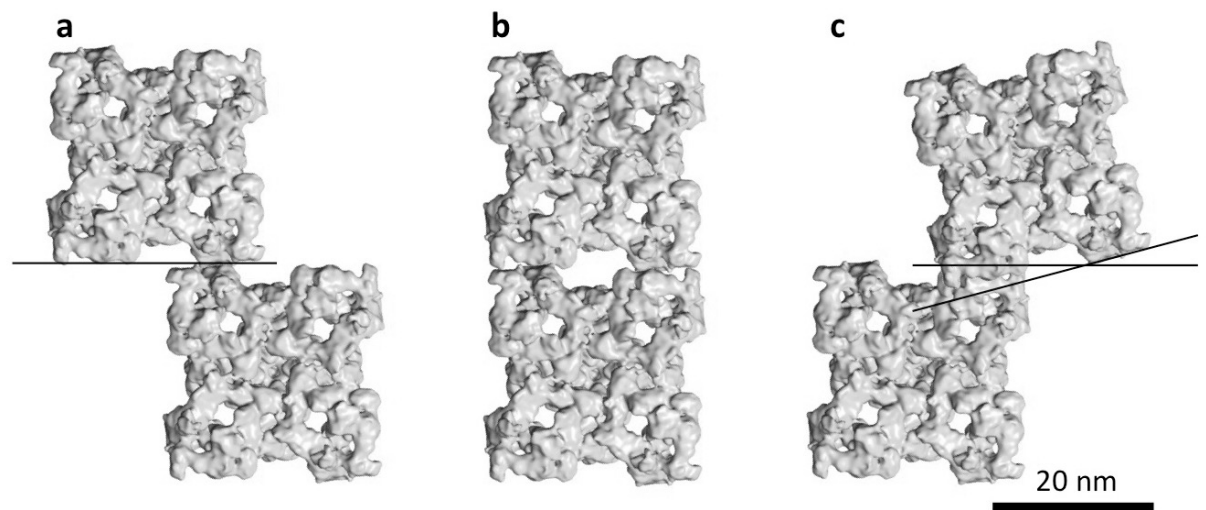

Figure 1. Different quaternary arrangements of ryanodine receptors. (a) Checkerboard, found in skeletal muscle. (b) Side-by-side, found in cardiac muscle. (c) Oblique, found in cardiac muscle. The lines indicate the angle between two neighboring channels.

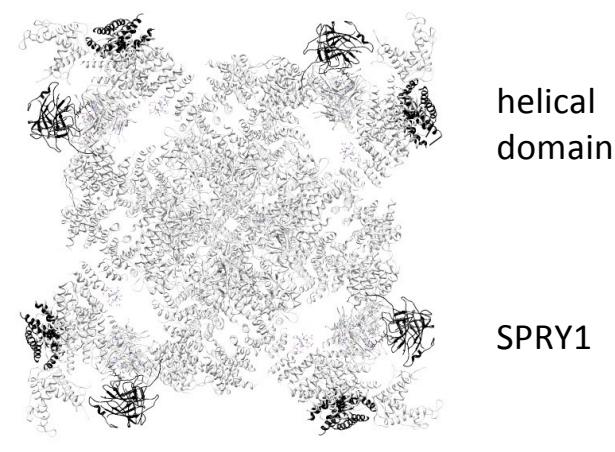

Figure 2. The two dimerization domains of RyR are highlighted in darker color in the atomic structure of RyR1 (3j8h.pdb). 